\title{
Principles of Structured Food Emulsions: \\ Novel formulations and trends
}

\author{
Schubert, H.; Engel, R.; Kempa, L. \\ University of Karlsruhe, Institute of Bio and Food Process Engineering, Fritz-Haber-Weg 2, \\ D-76131 Karlsruhe, Germany. \\ Email addresses: helmar.schubert@lvt.uni-karlsruhe.de; \\ robert.engel@1vt.uni-karlsruhe.de; lidia.kempa@1vt.uni-karlsruhe.de
}

\begin{abstract}
Most properties of food emulsions depend on emulsion microstructure, which is largely influenced by mean droplet diameter and droplet size distribution. The correlation between the properties and the microstructure is called property function. The process function describes the relationship between the microstructure and the process. If both functions are known, the properties of an emulsion may be derived directly from the process parameters. Although, for most food emulsions, the property functions are basically unknown, an example is discussed showing the functionality between important properties and the microstructure. The process function can be determined approximately by using the concept of energy density describing the relationship between mean droplet size and energy input per unit volume.

The droplet size of an emulsion depends on the intensity and mechanism of droplet disruption and on the extent of superimposed or subsequent droplet coalescence. Recent experiments have shown that the emulsifier, by reducing the interfacial tension, does not improve droplet disruption. The reason for this fact will be discussed and shown that only the interfacial tension of the unoccupied interface is relevant for droplet comminution. Thus the result of emulsification with the same apparatus at constant energy density is only determined by the time the emulsifier needs to stabilise the newly formed interface. A novel method to measure droplet coalescence during or directly after emulsification is presented.
\end{abstract}

Several methods suitable for the production of emulsions with the desired microstructure will be presented. Among these, newly developed valves inducing elongational flow have allowed for greatly increasing homogenisation efficiency in high pressure homogenisers and producing nanoemulsions with suitable emulsifier systems. Advances and new processes in emulsification with membranes and micro-structured systems as well as their potential will also be discussed.

Finally, an example demonstrates how nanoemulsions can be used to design functional foods with the help of the principles of structured food emulsions including the property and process functions. Phytosterols may significantly reduce cholesterol levels in humans, if they are appropriately formulated. Due to their poor solubility in water and oil, product engineering is required to achieve satisfactory dose-responses. Their interface-activity and tendency towards interface crystallisation further requires the application of certain emulsifier systems. With reference to cell culture studies on the bioavailability of carotenoids, a property function for phytosterol loaded emulsions can be derived exemplarily showing how the concept of process and property functions can be applied for formulation and quality improvement of a product using the principles of structured food emulsions.

\section{Production of emulsions}

Emulsions are systems made up of at least two practically immiscible liquids like e.g. oil and water, of which one liquid is finely dispersed in the other liquid. The dispersed liquid is also 
referred to as the disperse phase, whereas the other liquid is referred to as the continuous phase. The two basic types of emulsions are dispersions of a lipophilic or oil phase in a hydrophilic or watery phase or vice versa. With oil and water being the most common liquids for the preparation of food emulsions, these basic types of emulsions are referred to as oil-inwater- (o/w-) emulsions and water-in-oil- (w/o-) emulsions, respectively. More complex types consist of three or more phases, which can be achieved by e.g. dispersing a w/o-emulsion into a second watery phase, leading to a water-in-oil-in-water-(w/o/w-)emulsion. Due to the interfacial tension between the immiscible liquids, emulsions containing only oil and water are thermodynamically instable. By the use of surface active molecules, called emulsifiers in the following, emulsions can be kinetically stabilised. Furthermore, a difference in the densities of the two liquids may cause undesired creaming or sedimentation of the dispersed droplets. The occurrence of this physical instability can be delayed or prevented by increasing the viscosity of the continuous phase by so-called stabilisers, e.g. macromolecular substances. The basic types of emulsions as well as the role of emulsifiers and stabilisers are depicted in figure 1-1.

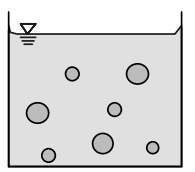

oil-in-water o/w

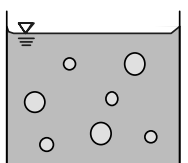

water-in-oil w/o

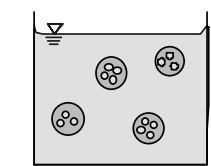

water-in-oilin-water (w/o)/w phase I:

hydrophilic liquid, e. g. water

phase II:

lipophilic liquid, e. g. oil

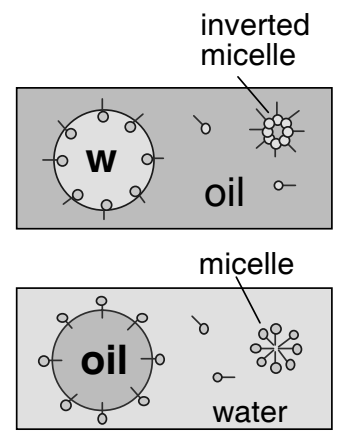

emulsifiers are surface active substances with hydrophilic and lipophilic groups

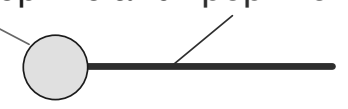

stabilizers are macromolecular substances, which increase the viscosity of the continuous phase.

Some substances (e. g. proteins) are both, emulsifier and stabilizer.

\section{Figure 1-1: Principle types of emulsions and emulsifying agents}

Most of the properties of emulsions depend on the emulsion microstructure, the emulsifiers used and the viscosity of the continuous phase. Microstructure is mainly a function of droplet size and droplet size distribution. Droplet size is of essential importance because of its great influence on physical and microbiological stability, rheological and optical characteristics, bioavailability or dose-response, taste and many other properties $(1,2)$. In many cases, emulsifying aims at producing droplets as fine as possible in such a way that the resulting emulsion is stable.

Fine emulsions can be produced in many different ways (2) as shown in figure 1-2. Mechanical processes are most frequently applied. They have been subject of recent publications $(3,4)$. Widely applicable rotor-stator systems are capable of producing emulsions both continuously and discontinuously. High-pressure systems, frequently referred to as highpressure homogenisers, are used to continuously produce fine-disperse emulsions. As in rotorstator systems, mechanical energy is the driving force for droplet disruption. In high-pressure systems, however, it is applied in the form of a pressure difference essential for droplet disruption. A simple orifice valve as shown in figure 1-2 has been found to be very efficient 
$(5,6)$. Its design is being modified and optimised at present with recently obtained results showing this system to be very promising (7).

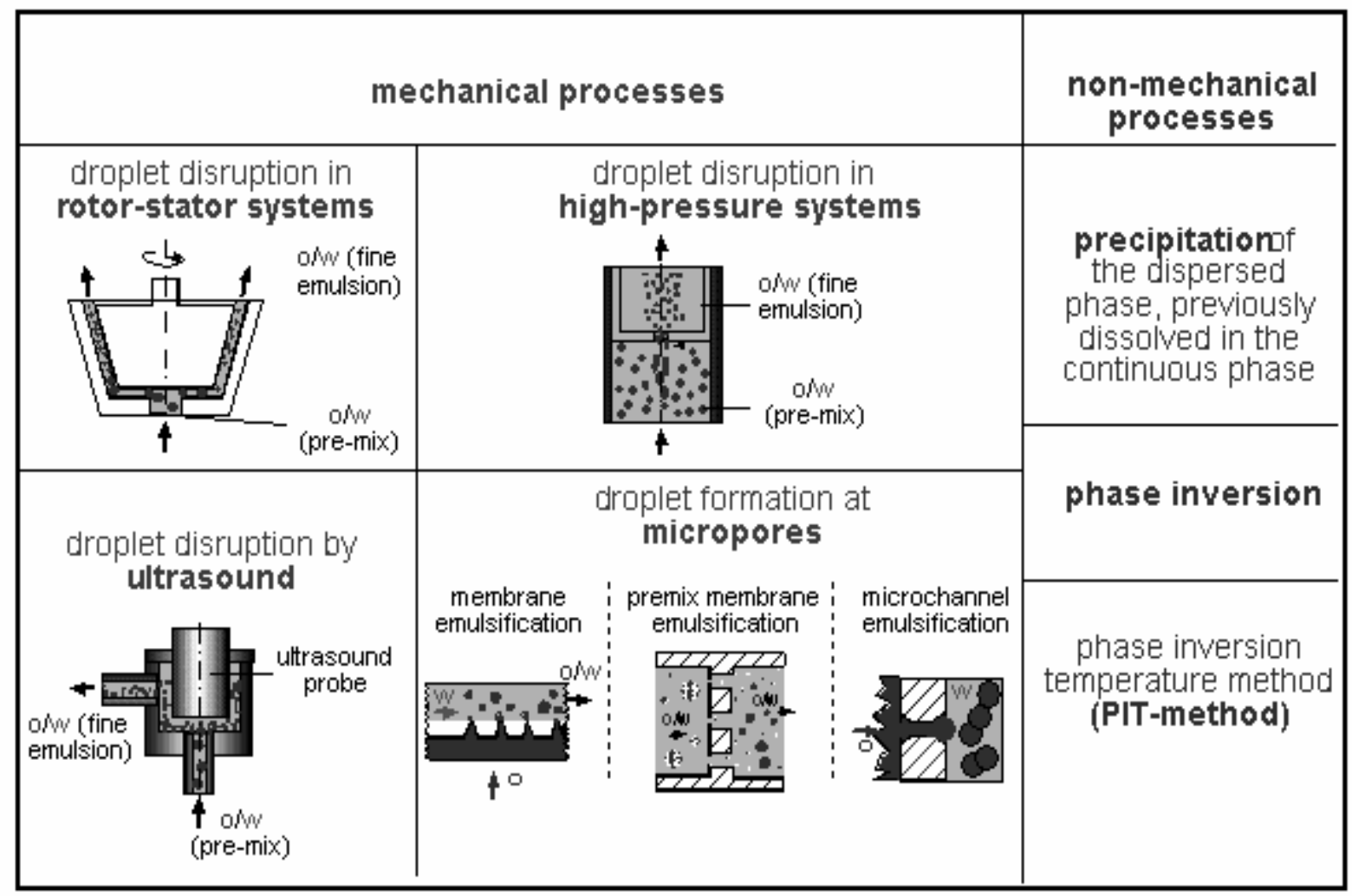

Figure 1-2: Overview of processes suitable for fine-emulsion formation

Fine-disperse emulsions can also be produced by ultrasound $(8,9,10)$. Until now, this method has mainly been employed in laboratories while wide droplet size distributions raise problems in continuous operation in industrial processes.

Besides the processes mentioned above, which are based on droplet disruption, emulsions may also be produced by droplet formation at membranes and micro-structured systems as depicted in figure 1-2, too. In this case, the disperse phase is forced through the micro pores of a membrane. The droplets forming at the pore outlets are detached by the flow of the continuous phase parallel to the membrane surface. The method developed by NAKASHIMA (11) in Japan in the 1990ies offers many new possibilities of gently producing very fine droplets and narrow droplet size distributions. Recent studies have shown that the process of forcing a coarse emulsion through membranes, known as premix membrane emulsification, is a means for achieving high throughputs, inducing phase inversions where desired and producing multiple emulsions $(12,13)$. At the authors' Institute, membrane emulsification has been a subject of intense studies $(14,15,16,17,18)$. Micro channel emulsification is a novel process reported upon recently to produce monodisperse emulsions $(19,20)$. A comparison of membrane systems and the micro-channel method is presented in figure 1-3.

Except for high-pressure and membrane or micro channel emulsification, emulsions may be produced either batch-wise or continuously. In the case of continuous emulsification, the ingredients are usually dosed separately and premixed in a stirrer. The resulting coarsedisperse raw emulsion is then fed into the droplet disruption machine for fine-emulsification. Energy input required for the formation of the raw emulsion is negligible compared to that 
required for fine emulsification. Single-step continuous processes excel at energy efficiency while discontinuous or multi-stage continuous ones usually allow the production of emulsions with narrower droplet size distributions.

\begin{tabular}{|c|c|c|c|c|}
\hline & 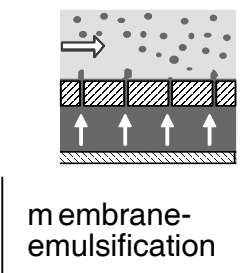 & 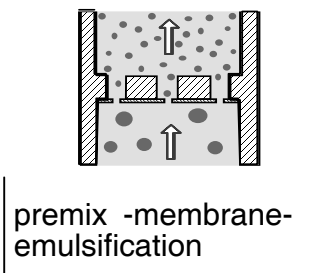 & $\begin{array}{l}\text { membrane jetting } \\
\text { emulsification }\end{array}$ & $\begin{array}{l}\text { microchannel- } \\
\text { emulsification }\end{array}$ \\
\hline $\begin{array}{l}\text { droplet detachment } \\
\text { (formation) by : }\end{array}$ & wall shear stress & flux & Rayleigh break-up & instability \\
\hline droplet size $\quad / \mu \mathrm{m}$ & $0.1-10$ & $0.1-10$ & $>0.2 ?$ & $>3$ \\
\hline $\begin{array}{l}\text { droplet size } \\
\text { distribution }\end{array}$ & narrow & narrow & narrow & monodisperse \\
\hline flux $/ \mathrm{m}^{3} / \mathrm{m}^{2} \mathrm{~h}$ & $\mathrm{~J}_{\mathrm{d}}<0.4(0.2)$ & $1<\mathrm{J}_{\mathrm{em}}<10(20)$ & $J_{d}>1$ & $\mathrm{~J}_{\mathrm{d}}<0.01$ \\
\hline $\begin{array}{l}\text { danger of } \\
\text { membrane fouling: }\end{array}$ & medium & high & low & low \\
\hline
\end{tabular}

Figure 1-3: Production of fine-emulsions using membranes or micro-structured systems

Besides these mechanical processes, there are several non-mechanical emulsifying processes applied to produce specific products for example in the chemical industry. One of these is based on the precipitation of the disperse phase previously dissolved in the external phase. Changes in the phase behaviour of the substances to be emulsified prompted by variation of temperature or composition or by mechanical stress are used to achieve the desirable dispersed state of the system. Another process of interest is the phase-inversion temperature (PIT) method discussed in (21).

Emulsions are most frequently produced in a continuous mode by mechanical droplet disruption. However, the principles prevailing differ depending on the type of mechanism used for droplet disruption. Fundamental work in this field has been done by WALSTRA and SMULDERS (22). Accordingly, figure 1-4 shows the maximum droplet size $\mathrm{x}_{\max }$ resulting from different types of stress in turbulent and laminar flow under the influence of inertia, shear or expansion forces. The table, which differentiates among rotor-stator systems, ultrasound systems and high-pressure homogenisers, also shows the different Reynolds numbers $\mathrm{Re}_{\text {flow }}$ of the continuous phase and $\mathrm{Re}_{\text {droplet }}$ of the flow around droplets: in $\mathrm{Re}_{\text {droplet }}$, the droplet diameter is considered the characteristic length. It becomes obvious that the maximum droplet size depends on both power density $\mathrm{P}_{\mathrm{V}}$ and residence time $\mathrm{t}$. The concept of energy density is based on these functions $(23,24)$. Experiments have shown a simple relationship to exist between maximum droplet size and the Sauter diameter $\mathrm{x}_{3,2}$ (mean droplet size of the droplet collective) (25). The maximum droplet size shown in figure 1-4 may hence be replaced by the Sauter diameter, if the respective proportionality factor is adequately adjusted. In high-pressure homogenisers, the Sauter diameter is a direct function of energy density $\mathrm{E}_{\mathrm{V}}$ that equals the effective pressure difference $\Delta \mathrm{p}$ at the homogenising valve. In rotor-stator systems and ultrasound homogenisers, Sauter diameter and energy density are only linked by approximations on the basis of experimental data as noted in figure 1-4. 


\begin{tabular}{|c|c|c|c|}
\hline & $\begin{array}{l}\text { TURBULENT } \\
\text { inertia forces }\end{array}$ & $\begin{array}{l}\text { TURBULENT } \\
\text { shear forces }\end{array}$ & $\begin{array}{c}\text { LAMINAR } \\
\text { shear or stretch forces }\end{array}$ \\
\hline $\mathrm{Re}_{\text {flow }}$ & $>2000$ & $>2000$ & $<1000$ \\
\hline $\mathbf{R e}_{\text {droplet }}$ & $>1$ & $<1$ & $<1$ \\
\hline \multirow{3}{*}{$\begin{array}{l}\text { rotor-stator- } \\
\text { mill, } \\
\text { ultrasound- } \\
\text { hom ogeniser }\end{array}$} & $x_{\max } \propto P_{V}^{-0,4}$ & $x_{\max } \propto P_{V^{-0,5}}$ & \multirow{3}{*}{$\left.x_{\max } \cong A_{3} E_{V^{-0,4 * *}}\right)$} \\
\hline & $\left.\mathbf{x}_{\max } \propto \mathbf{t}^{-0,3 *}\right)$ & $\left.x_{\max } \propto t^{-0,3 *}\right)$ & \\
\hline & $\left.\mathrm{x}_{\max } \cong \mathrm{A}_{1} \mathrm{E}_{\mathrm{V}}^{-0,35 * \pi}\right)$ & $\left.x_{\max } \cong A_{2} E_{V}^{-0,75 * *}\right)$ & \\
\hline $\begin{array}{l}\text { high-pressure } \\
\text { hom ogeniser }\end{array}$ & $\mathrm{x}_{\max }=\mathrm{A}_{4} \mathrm{E}_{\mathrm{V}}^{-0,6}$ & $x_{\max }=A_{5} E_{V}-0,75$ & $x_{\max }=A_{6} E_{V}{ }^{-1}$ \\
\hline
\end{tabular}

\begin{tabular}{|c|c|c|}
\hline In general: & $x_{3,2}=$ sauter diam eter & ${ }^{*}$ ) exponent from experiments \\
\hline$x_{3,2}=A_{i} E_{V}^{-b}$ & $E_{V}=$ energy density $\left[\mathrm{J} / \mathrm{m}^{3}\right]$ & ${ }^{*}$ ) approximated exponent \\
\hline
\end{tabular}

Figure 1-4: Mechanisms of droplet disruption in continuous emulsification

\section{The concept of energy density of emulsions}

In general, the Sauter diameter in continuous emulsification with droplet disruption may be described by the relation

$x_{3,2}=a_{i} \cdot E_{v}{ }^{-b}$

with the constants $a_{i}$ and $b$ depending on the emulsifying agents, the properties of the continuous and the disperse phase and the emulsification equipment applied.

As mentioned above (23), some secondary conditions must be fulfilled to make sure this relation applies. First of all, the forces at the droplet surface must exceed a critical threshold above which droplets are disrupted. Furthermore, the time allowed for droplet deformation must exceed a critical deformation time in order to deform droplets to an extent sufficient for break up. This means that a critical power density $\mathrm{P}_{\mathrm{v}}$ must be exceeded for droplets to be disrupted.

The concept of energy density (23) according to equation (2.2) developed at our Institute has been found to be a useful approximation for mechanical emulsification practice. It is applied to design, scale-up and control of continuously operating emulsifying apparatus. Energy density

$E_{V}=\frac{P}{\dot{V}}=\Delta p$

is easy to measure because in an emulsifying system, power input $\mathrm{P}$ into the machine, volume flow rate $\dot{V}=\mathrm{dV} / \mathrm{dt}$ of the emulsion and pressure difference $\Delta \mathrm{p}$, in case of a high-pressure homogeniser, are easily accessible. The concept of energy density may also be applied to compare different mechanical emulsification processes. An example of such a comparison is shown in figure 2-1. In membrane emulsification, mean droplet sizes (Sauter diameters) not only depend on energy density, but also on volume proportion $\varphi$ of the disperse phase (16). The comparison illustrates that for equal energy densities, different types of emulsification equipment yield a wide range of different droplet sizes. In the comparison presented here, 
droplet coalescence, which was minimized by choosing adequate emulsifiers in the experiments, has been ignored. Droplet coalescence during emulsification will be discussed separately in chapter 4 .

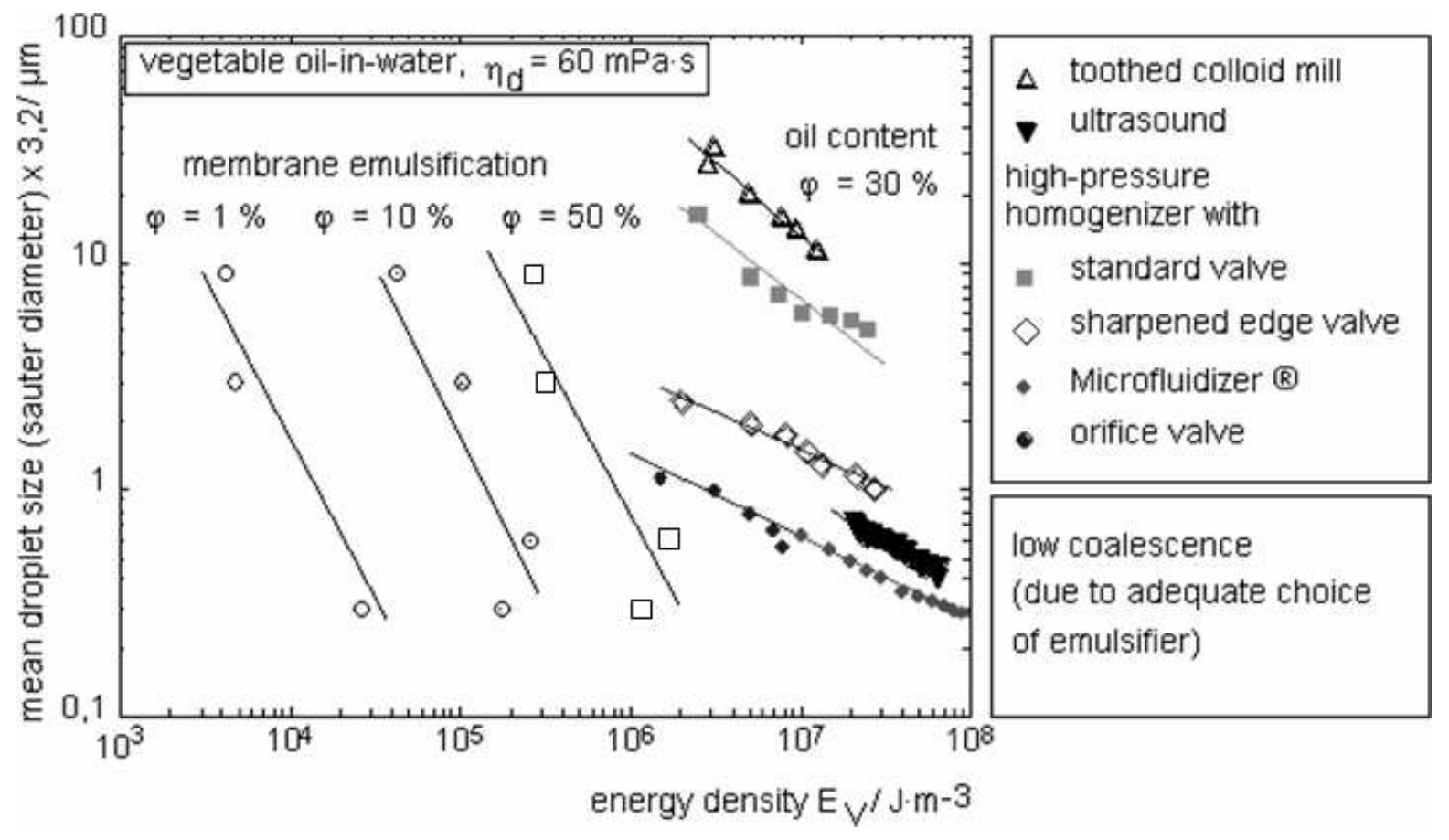

Figure 2-1: Comparison of various homogenization processes by means of energy density

Another important factor for homogenisation of emulsions is the ratio of the viscosity of the disperse phase $\eta_{d}$ to that of the continuous phase $\eta_{c}$. A comparison of the results of homogenizing an o/w-emulsion with a high ratio $\eta_{\mathrm{d}} / \eta_{\mathrm{c}}$ in different high pressure homogenization valves is shown in figure 2-2. This comparison clearly shows a great advantage in terms of energy required for homogenization as well as the result regarding droplet sizes for a simple orifice valve. These results can be explained by the three dimensional elongational flow in front of an orifice valve which results in deformation of the droplets to filaments, which will then be broken up by inertial forces and instabilities caused by the turbulent flow regime behind the orifice valve.

Besides the viscosity of the disperse phase, the interfacial tension between the continuous and the disperse phase plays an important role in hindering droplet disruption. The minimal required energy for droplet disruption is directly proportional to the resulting increase in interfacial area as well as the interfacial tension between the two phases. Therefore, reducing the interfacial tension by the addition of emulsifier and its absorption to the interface before homogenisation has long been considered a means for improving homogenisation efficiency by facilitating droplet disruption. In order to investigate the emulsifier's effect on droplet disruption and homogenisation efficiency, it was the aim to allow for investigating droplet disruption separately from droplet coalescence. For this purpose, a special homogenisation valve for high pressure homogenisation was designed and applied in experiments with various emulsion systems. A schematic drawing of the valve is provided in figure 2-3 (26). This valve allows for adding the emulsifier to the emulsion immediately as well as at various distances behind the outlet of the valve. The results of these investigations show that the emulsifier neither has influence on droplet deformation before the valve, nor on the following droplet 


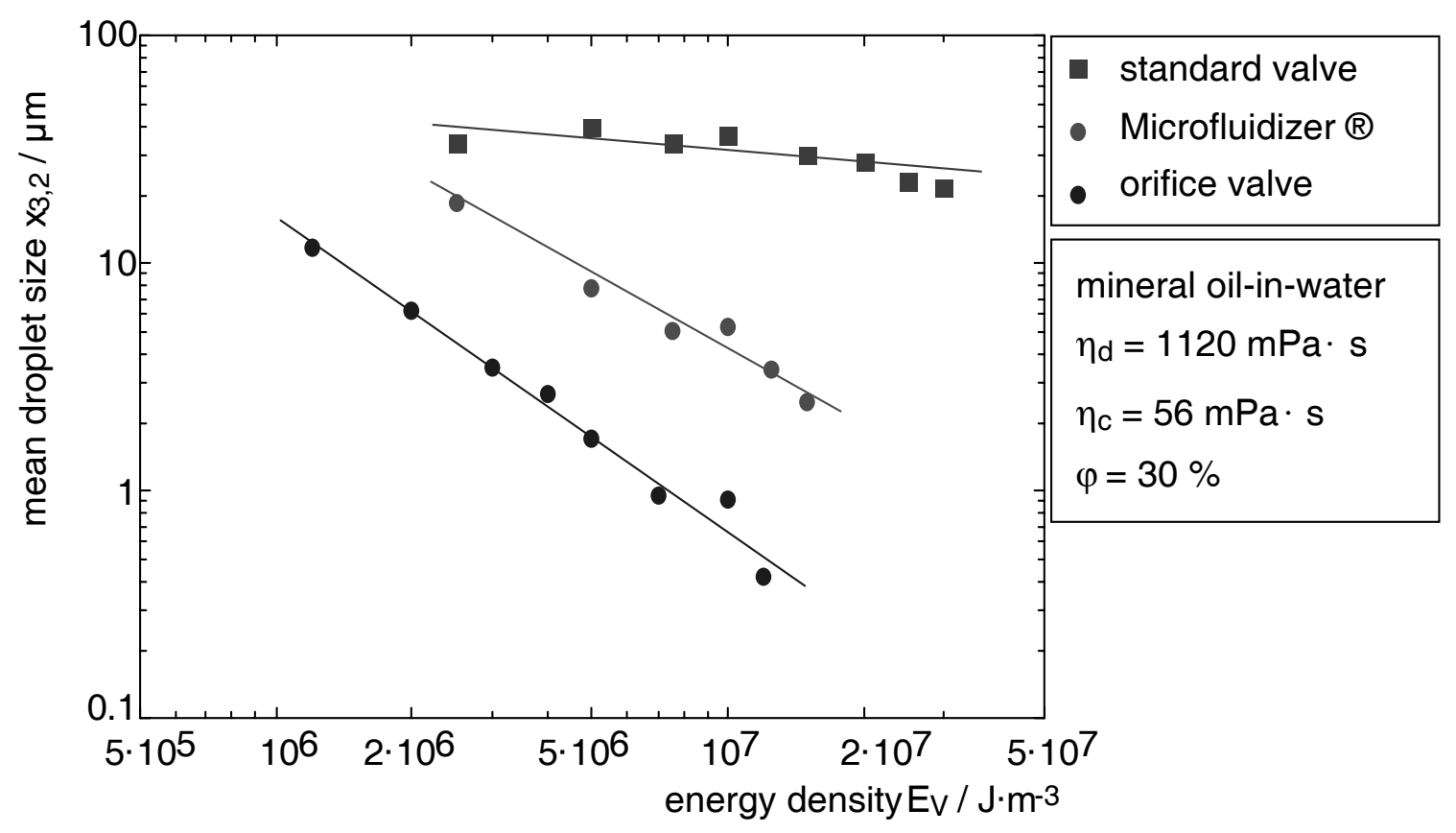

Figure 2-2: Comparison of homogenisation efficiency of various homogenisation valves for an o/w-emulsion with a high viscosity ratio $\eta_{\mathrm{d}} / \eta_{\mathrm{c}}$.

disruption. This can be clearly seen in figure $2-4$, where the mean droplet diameters in emulsions homogenised with and without emulsifier present in the pre-emulsion are shown. It also shows that adding the emulsifier too far behind the valve causes droplet coalescence due to the interfacial area not being covered by emulsifier molecules and the droplets thus being unprotected against coalescence (26). The same holds for emulsifiers like e.g. protein isolates which do not absorb to the interface quickly enough to protect the droplets form coalescence, although parts of this effect may be compensated by increasing the emulsifier concentration.

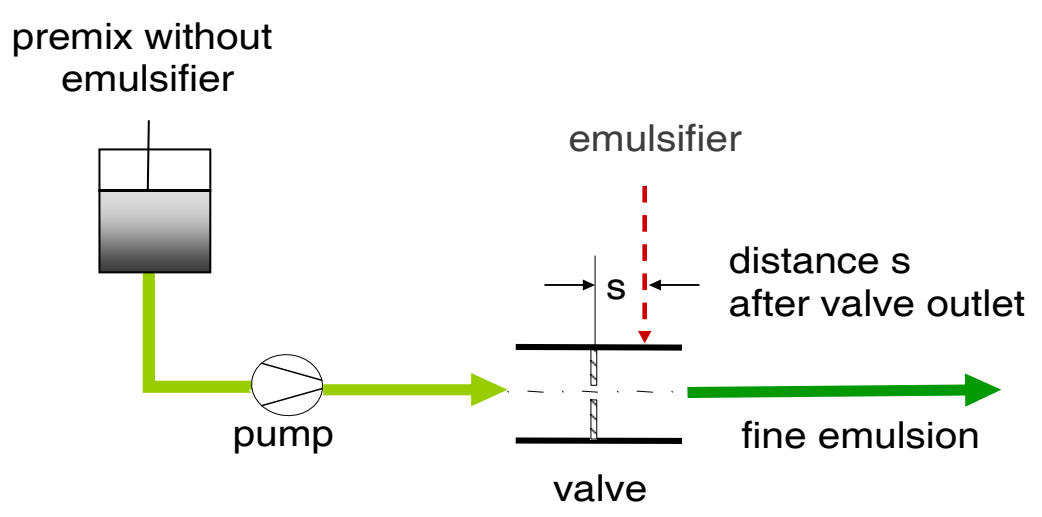

Figure 2-3: Schematic drawing of the high pressure homogenisation valve allowing for addition of the emulsifier at various distances behind the valve

\section{$3 \quad$ Adjustment of emulsion properties}

The results presented so far provide a basis for product design and the formulation of emulsions. In this context, product design means creating a product with certain desired 
properties that are adjusted by engineering methods. As it is very expensive to vary all essential parameters of a technical apparatus in order to ultimately achieving the desired property of a product, an interim attribute is introduced. In the case of emulsions, their microstructure is taken for the interim attribute, which is mainly determined by mean droplet size, droplet size distribution, and type of emulsifier and stabiliser. For most problems, it may be sufficient to simply characterise the microstructure by the mean droplet size, a physical value easily measurable and adjustable by means of emulsifying equipment.

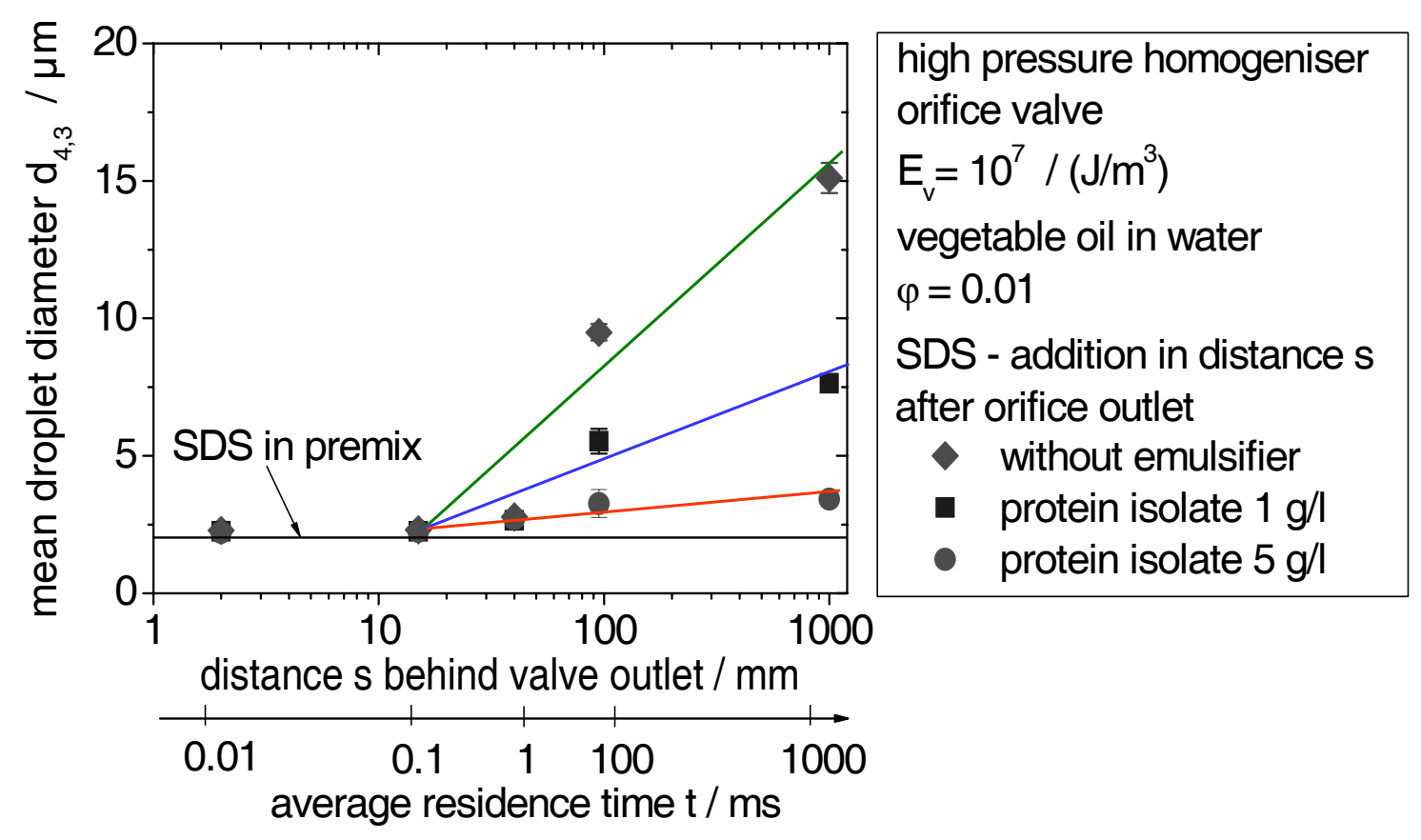

\section{Figure 2-4: Mean droplet diameters in emulsions homogenised with and without the presence of an emulsifier and different localisation for addition of emulsifier behind the homogenising valve}

The link between the emulsion's microstructure and the process or emulsifying apparatus, respectively, is called process function (27). In the present simplified case, the process function can be described by equation (2.1). The mean droplet size, accordingly, depends on the energy density, which can either be directly determined or taken from figure 2-1 for the different apparatus. Linking microstructure and emulsion properties has been found to be much more complex. According to RUMPF, this link is called property function (28). In many cases it is difficult to define this property function, i.e. to link product properties and physical characteristics (microstructure). Therefore, in most cases, the property function is a far more complex equation and rather stands for a complex connection between properties and microstructure. Figure 3-1 illustrates the links among emulsion properties, microstructure and process parameters. The concept of product design and formulation is currently in the process of further improvement. In the case of emulsions, the process function provides a useful approximation, while property functions for most applications, remain to be developed (see $(3,4))$. If in the present cases of carotenoid or phytosterol formulations dose response was merely a function of droplet size, it would be easy to derive a property function. Process and property function would then provide the possibility to achieve the desired properties by selecting the appropriate process. The current investigations will show if, and if so, to what extent, this is a practicable way. 


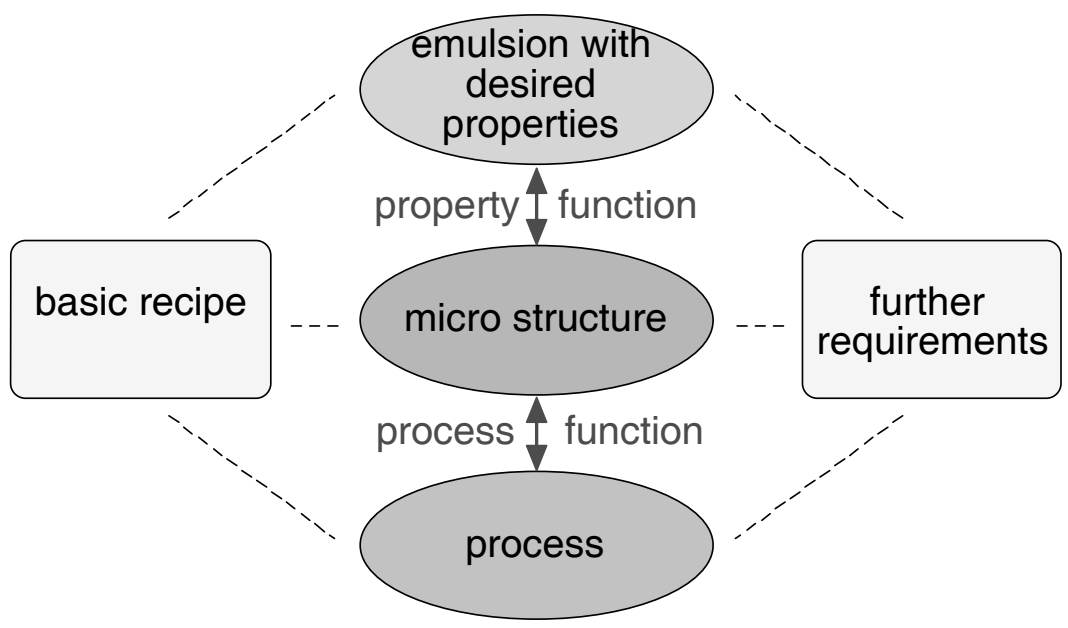

\section{Figure 3-1: Achieving a product with desired properties by taking into account the micro structure, the process and property functions as well as the basic recipe and certain boundary conditions}

\section{$4 \quad$ Stability of emulsions}

Without doubt, stability is the most important property of emulsions. However, it has to be differentiated between physical, chemical and microbiological stability, of which only the first will be subject to detailed discussion here. An emulsion is called physically stable if its dispersed state does not change, i.e. if its droplet size distribution remains constant regardless of time or volume element observed. First of all, droplets must not sediment, aggregate or coalesce; changes in droplet sizes due to Ostwald ripening, i.e. the growth of large droplets at the expense of small ones due to different capillary pressures, or phase inversion are not admissible either. Emulsions with sufficiently small droplets, which are prevented from aggregating or coalescing by the use of suitable emulsifiers, exhibit great physical stability. In many cases, Ostwald ripening is controlled by diffusion. Because the various liquid phases of an emulsion are usually poorly soluble in each other, Ostwald ripening mostly is of minor importance. However, if Ostwald ripening is to be minimised, this undesirable process is slowed down by producing droplets as equal in size as possible thereby reducing the differences in capillary pressures.

The chemical stability of an emulsion reflects its resistance against chemical changes. Mostly oxidation of fats and oils is the critical reaction for chemical deterioration of emulsions. The addition of antioxidants and protection against external influences such as light or excessive heat, as well as suitable diffusion barriers on the surfaces between the different liquid phases, improve the chemical stability.

The physical stability of emulsions during and immediately after emulsification, which we have called short-term stability, presents specific problems, too. The short-term stability of an emulsion indicates whether the newly formed droplets of an emulsion are sufficiently protected against coalescence during or directly after emulsification. Therefore, it decides on success or failure of the emulsification process because mechanical emulsification processes are expected to not only size-reduce, but also to immediately stabilise these smaller droplets. Droplets successfully protected against coalescence remain small, resulting in the desired fine-disperse emulsion. If coalescence cannot be successfully prevented, droplets will flow together and form larger droplets. In a critical case, this could completely reverse the previous size reduction. In unfavourable cases, it may even cause emulsions to break. Therefore, 
avoiding droplet coalescence during and immediately following the formation of new droplets, i.e. achieving sufficient short-time stability, is of utmost importance for any emulsification process.

Despite many indications for droplet coalescence in emulsifying apparatus, this question is discussed controversially in the pertinent literature. Based on the Gibbs-Marangoni effect, some authors hold the view that newly formed droplets in emulsions with sufficient cannot coalesce with an emulsifier present. For immediate detection of droplet coalescence during emulsification, a method has been developed at our Institute by DANNER (29) that he called colouring method. According to his work, the droplets of two identical raw emulsions are stained with different colours. Thereafter, a fine emulsion is produced from the mixture of these two raw emulsions involving disruption of the large stained droplets. The colours have been selected in such a way that a third colour can only result from coalescence of differently coloured droplets and the subsequent mixture of their colorants, but not from diffusion. The presence of droplets of this third colour after emulsification indicates that coalescence has occurred. Assays of the fraction of the droplets of the third colour at different times of a batch-wise emulsification process allow for quantification of the extent of coalescence. The principle of this method is shown in figure 4-1. The coalescence of white and black droplets results in droplets of the third colour grey. After a short emulsification time $t_{1}$, only a few droplets have coalesced and therefore are grey, whereas after an extended emulsification time $t_{2}$, nearly all droplets have been subject to coalescence at least once. By the application of an image-evaluating software, reliable and reproducible data of droplet coalescence during emulsification are obtained (29).

DANNER (29) developed a stochastic model in order to describe the processes of coalescence in emulsions in greater detail. Provided the frequency of droplet collision is known, both probability and rate of coalescence may be calculated. In this way, coalescence in different types of emulsifying equipment can be monitored quantitatively. In figure 4-2, results of emulsions with different emulsifiers are presented exemplarily. The area covered by droplets of the third, mixed colour is plotted over the emulsification time. In the present case, the droplets of the two raw emulsions were stained blue and yellow, respectively, with coalesced droplets turning green. The figure reveals different processes of coalescence depending of the application of Tween ${ }^{\circledR} 80$ or egg yolk as emulsifier. Tween ${ }^{\circledR} 80$ is shown to provide a much better short-term stability than egg yolk. The colouring method hence is a very useful and practice-oriented test to judge the stabilising efficiency of different emulsifiers and stabilisers. It may also serve for studying further essential parameters influencing droplet coalescence during and immediately after emulsification. Finally, the method has also been found helpful by those investigating possibilities of stabilising emulsions against droplet coalescence hydrodynamically by trying to develop stabilising zones (30) immediately following the area of size-reduction (29).

\section{Formulation of emulsions containing poorly soluble compounds}

Some compounds with interesting properties for food, feed or pharmaceuticals are insoluble in water and insufficiently soluble in edible oils at room temperature. Among these are some carotenoids with health-promoting properties, phytosterols with their cholesterol-lowering and anticarcinogenic effects, as well as the majority of novel pharmaceutically active substances. Because of insolubility or poor solubility of their usually crystalline form, they are barely bioavailable and have poor dose responses. In order to improve bioavailability and 

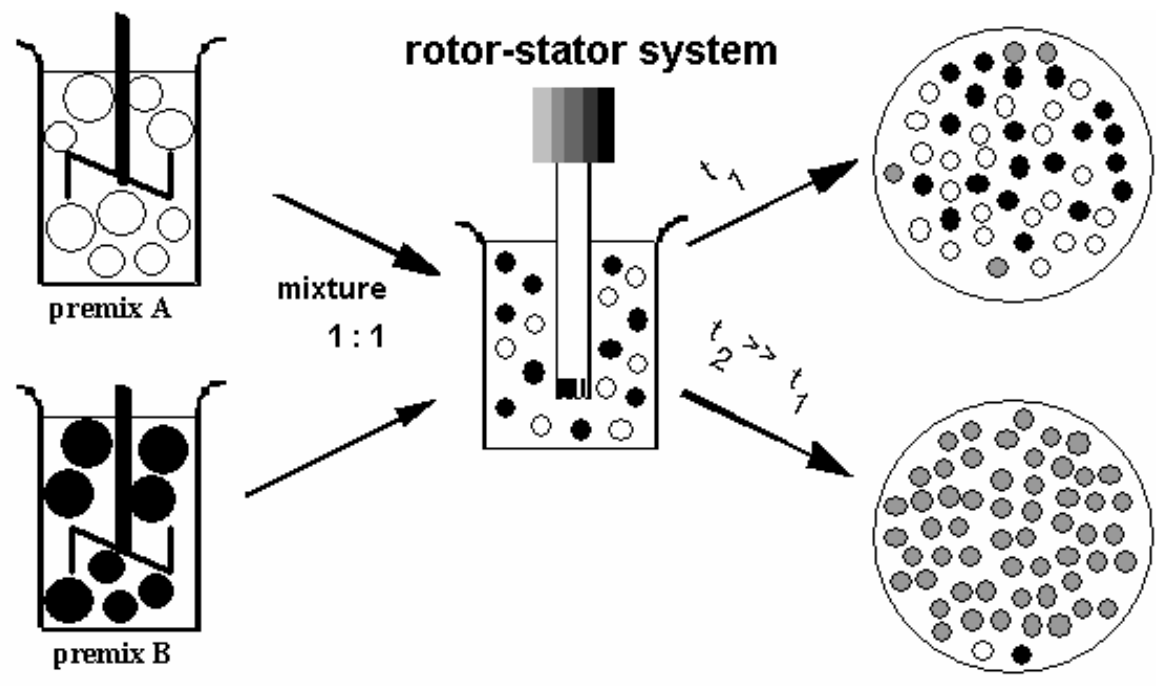

Figure 4-1: The principle of the couloring-method for determining the occurrence of and rate of coalescence

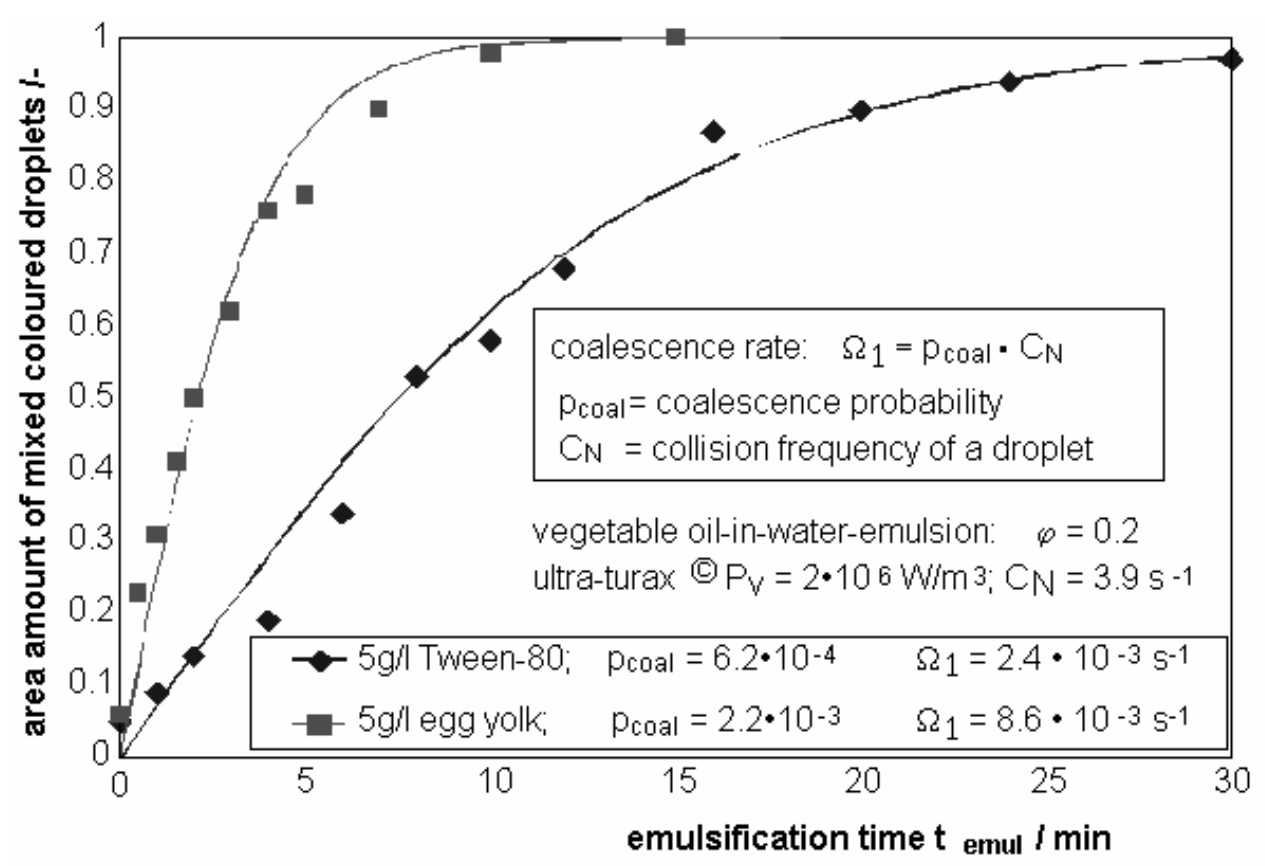

Figure 4-2: Increase of coalescence with emulsification time measured by the colouring method for emulsions prepared with different emulsifiers

dose response, products containing these compounds may be formulated as emulsions (31) in which the poorly soluble compound is present in the dispersed lipid phase at a highly supersaturated concentration. Provided the droplets of the supersaturated oil phase are sufficiently small, carotenoids and, to some extend, phytosterols, for instance, can be stabilized in a solubilised state at a supersaturated concentration for a sufficiently long period of time.

The procedure of producing Phytosterol loaded o/w-emulsions is schematically shown in figure 5-1. The two phases of the emulsion are prepared separately. A maximum of $35 \%$ of 
phytosterols, referring to the disperse phase, could be solved in a medium chain triglyceride (MCT-oil) together with an oil soluble emulsifier at approximately $100{ }^{\circ} \mathrm{C}$. Demineralised water with Tween ${ }^{\circledR} 20$ as emulsifier at a concentration of 1 weight percent of the total emulsion was heated to $90{ }^{\circ} \mathrm{C}$. The oil soluble emulsifier, e.g. a lecithin, allows for even higher supersaturation of solubilised phytosterols in the dispersed oil phase and further more prevents immediate crystallisation during the emulsification process. The two hot phases were then transferred into the sample inlet vessel of a high-pressure homogeniser with both devices kept at $90{ }^{\circ} \mathrm{C}$ by a water bath and intensively stirred.

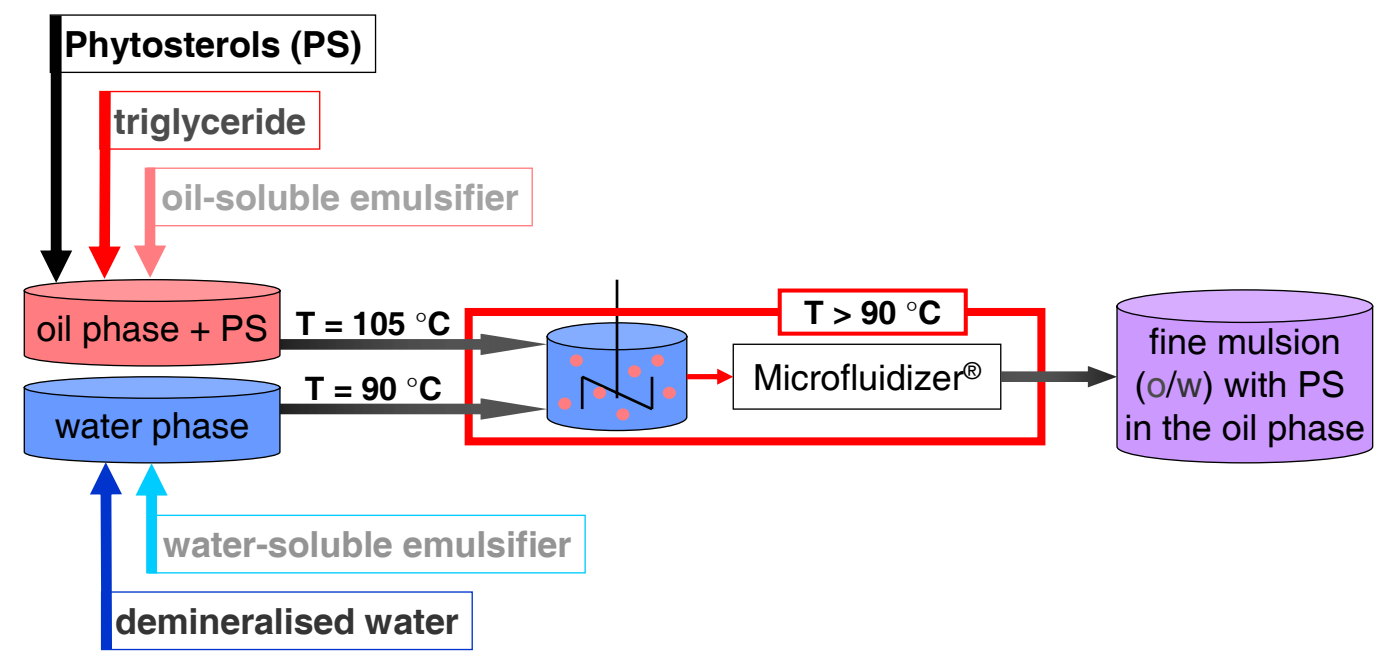

\section{Figure 5-1: Process fort he production of Phytosterol loaded o/w-emulsions}

The resulting coarse raw emulsion was homogenised at a pressure of 1000 bar at $90{ }^{\circ} \mathrm{C}$. After emulsification, the stable fine-disperse emulsion with a Sauter diameter well below $1 \mu \mathrm{m}$ could cool down to room temperature.

Since phytosterols, due to their molecular structure, are interface active components, they tend to migrate to and crystallise at the oil-water interface of the oil droplets in O/W emulsions. In order to suspend this effect on the emulsion's short-term stability, an emulsifier system of one oil- and one water-soluble and fast stabilising emulsifier such as Tween ${ }^{\circledR} 20$ had to be employed.

Regarding their physical stability, the small Sauter diameter of the approximately ten-times phytosterol-supersaturated oil droplets together with the crystallisation inhibitor make sure that no crystallisation or creaming occurs.

The formulation of emulsions enriched with poorly soluble compounds such as phytosterols or carotenoids may - in a greatly simplified way - be demonstrated by means of the process and property functions. Following equation (2.1), the process function for emulsions produced in a high pressure homogeniser with an orifice valve or in a Microfluidizer ${ }^{\circledR}$ with a homogenising pressure of $\Delta \mathrm{p}=\mathrm{E}_{\mathrm{V}}$, is (33)

$\mathrm{x}_{3,2}=4,5 \cdot 10^{-3} \Delta \mathrm{p}^{-0,5}$

with a resulting Sauter diameter $\mathrm{x}_{3,2}$ in $\mathrm{m}$ and a given effective pressure difference $\Delta \mathrm{p}$ in $\mathrm{Pa}$.

Above that, it can be assumed that the bioavailability B of the added compound, e.g. phytosterols, increases with decreasing Sauter diameter. The validity of this interrelationship could be demonstrated for example by AX ET AL. (31) for carotenoids. As possible values of B are in a range between $B_{\min }$ and $B_{\max }, B$ and $x_{3,2}$ may be linked by the simple equation 


$$
\mathrm{B}=\frac{\mathrm{B}_{\max }+\mathrm{B}_{\min } \cdot \mathrm{k} \cdot \mathrm{x}_{3,2}}{1+\mathrm{k} \cdot \mathrm{x}_{3,2}}
$$

For the border cases $\mathrm{B}_{\min }=0$ and $\mathrm{B}_{\max }=1$, equation (5.2) reduces to

$$
\mathrm{B}=\frac{1}{1+\mathrm{k} \cdot \mathrm{x}_{3,2}}
$$

For this case, equations (5.2) and (5.3), resp., represent the applicable property function. The constant values $B_{\min }, B_{\max }$ and $\mathrm{k}$ must be derived by experiment, for instance, from human studies. Until now, the equations for this property function have only been verified in in-vitro tests for carotenoids. Therefore, precise values for the constant required cannot yet be derived from appropriate experiments. However, to illustrate the procedure of developing emulsions by making use of the process and property functions, it is assumed that $\mathrm{k}=1 \mu \mathrm{m}^{-1}=10^{6} \mathrm{~m}^{-1}$. Accordingly, the following equation results from equations (5.1) and (5.3):

$$
\frac{\Delta \mathrm{p}}{[\mathrm{Pa}]}=\left(\frac{4,5 \cdot 10^{-3} \cdot \mathrm{k} \cdot \mathrm{B} \cdot[\mathrm{m}]}{1-\mathrm{B}}\right)^{2}
$$

If, for instance, the bioavailability of the incorporated compound shall be $60 \%(\mathrm{~B}=0,6)$, a homogenising pressure of $\Delta \mathrm{p} \approx 450$ bar is needed to produce the desired emulsion. The example elucidates - even if some assumptions and substantial simplifications have been made - how useful process and property functions are, which, however, may be much more complex in many cases. Usually, some secondary conditions have to be considered as well. For example, in the present case, the aforementioned stability of the resulting emulsion and the chemical stability of the incorporated compound would have to be taken into account, too (31).

\section{Acknowledgement}

The studies were financially supported by Deutsche Forschungsgemeinschaft (DFG) within the joint research project "Lipide und Phytosterole in der Ernährung" and within the framework of the DFG Graduiertenkolleg 366 "Grenzflächenphänomene in aquatischen Systemen und wässrigen Phasen". The authors would also like to thank the "Bundesministerium für Bildung und Forschung" for the financial support of the subproject 0312248A/7 "Synthese und Formulierung von Carotinoiden".

\section{$7 \quad$ References}

1. Schubert, H. (Ed.); 2005: Emulgiertechnik - Grundlagen, Verfahren und Anwendungen. B. Behr`s Verlag, Hamburg/Germany.

2. Schubert, H.; 2005: Einführung in die Emulgiertechnik. In (1), p. 1-15.

3. Schubert, H.; 2003: Neue Entwicklungen auf dem Gebiet der Emulgiertechnik. In: Kraume, M.(Ed.); 2003: Mischen und Rühren. Wiley-VCH Verlag, Weinheim/Germany, p.313-342.

4. Schubert, H. and Ax, K.; 2001: Verbesserung der gesundheitlichen Qualität von Lebensmitteln durch Erhöhung und Modifikation des Carotinoidgehaltes. 59. 
Diskussionstagung, Forschungskreis der Ernährungsindustrie e.V. (FEI), Bonn/Germany, p. 106-137.

5. Stang, M.; Schuchmann, H. and Schubert, H.; 2001: Emulsification in high-pressure homoginizers. Eng. Life Sci. 1, 4, p. 151-157.

6. Tesch, S.; Freudig, B.; Schubert, H.; 2002: Herstellen von Emulsionen in Hochdruckhomogenisatoren, Teil 1. Chemie Ingenieur Technik 74, p. 875-880.

7. Freudig, B., Tesch, S., and Schubert, H.; 2002: Herstellen von Emulsionen in Hochdruckhomogenisatoren, Teil 2. Chemie Ingenieur Technik 74, p. 880-884.

8. Behrend, O.; Ax, K.; Schubert, H.; 2000: Influence of continuous phase viscosity on emulsification by ultrasound. Ultrasonics Sonochemistry 7, p. 77-85.

9. Behrend, O.; Schubert, H.; 2001: Influence of hydrostatic pressure and gas content on continuous ultrasound emulsification. Ultrasonics Sonochemistry 8, p. 271-276.

10. Behrend, O.; 2002: Mechanisches Emulgieren mit Ultraschall. Dissertation University of Karlsruhe, GCA-Verlag Herdecke/Germany.

11. Nakashima, T.; et al.; 1994: Development of membrane emulsification and its applications to food industries. Shokuhin Kogyo Gakkaishi 41, p. 70-76.

12. Suzuki, K.; 2000: Membrane emulsification for food processings. Proc. NFRI-BRAIN Workshop, Oct. 16-17, Tsukuba/Japan, p. 635-636.

13. Vladisavljevic, G. T.; Shimizu, M. and Nakashima, T.; 2005: Preparation of uniform multiple emulsions using multi-stage premix membrane emulsificatin technique. In (1), p. $433-468$.

14. Altenach-Rehm, J., Schubert, H. and Suzuki, K; 2002: Premix Membranemulgieren mittels hydrophiler und hydrophober PFTE-Membranen zur Herstellung von O/WEmulsionen. Chemie Ingenieur Technik 74, p. 587-588.

15. Schröder, V. and Schubert, H.; 1999: Influence of emulsifier and pore size on membrane emulsification. In: Food Emulsiones and Foams. Royal Society of Chemistry, Cambridge/UK.

16. Schröder, V.; 1999: Herstellen von Öl-in-Wasser-Emulsionen mit mikroporösen Membranen. Dissertation University of Karlsruhe, Shaker Verlag Aachen/Germany.

17. Vladisavljevic, G. T.; Tesch, S.; 2002: Preparation of water-in-oil emulsions using microporous polypropylene hollow fibers. Chemical engineering and Processing 41, S. 231-238.

18. Lambrich, U.; Schröder, V. and Vladisavljevic, G. T.; 2005: Emulgieren mit mikroporösen Systemen. In (1), p. 369-431. 
19. Nakajima, M.; 2000: Microchannel emulsification for monodispersed microspheres. Proc. NFRI-BRAIN Workshop, Oct.16-17, Tsukuba/Japan, p. 626-628.

20. Nakajima, M. and Kobayashi, I.; 2001: Emulsification with newly fabricated throughtype microchannel. 11th World Congress of Food Science and Technology, April 22-27, Seoul/Korea.

21. von Rybinski, W.;2005: Herstellen von Emulsionen nach der PhaseninversionsMethode. In (1), p. 469-485.

22. Walstra, P. and Smulders, P. E. A.; 1998: Emulsion Formation. In: Modern aspects of emulsion science. Royal Society of Chemistry, Cambridge/UK.

23. Karbstein, H.; 1994: Untersuchungen zum Herstellen und Stabilisieren von O/W Emuldionen. Dissertation University of Karlsruhe.

24. Schubert, H. and Karbstein, H.; 1994: Mechanical emulsification. In: Development in Food Engineering, Part 1. Blacky Academic \& Professional, London.

25. Armbruster, H.;1990: Untersuchungen zum kontinuierlichen Emulgierprozess in Kolloidmühlen. Dissertation University of Karlsruhe.

26. Kempa, L.; Schuchmann, H.P. and Schubert, H.; 2006: Tropfenzerkleinerung und Tropfenkoaleszenz beim mechanischen Emulgieren mit Hochdruckhomogenisatoren. Chemie Ingenieur Technik 78, p. 765-768.

27. Krekel, J.; Polke, R.; 1992: Qualitätssicherung bei der Verfahrensentwicklung. Chemie Ingenieur Technik 64, p. 528-535.

28. Rumpf, H.; 1967: Über die Eigenschaften von Nutzstäuben. Staub-Reinhaltung der Luft 27, p. 70-76.

29. Danner, T.; 2001: Tropfenkoaleszenz in Emulsionen. Dissertation University of Karlsruhe, GCA-Verlag Herdecke/Germany.

30. Stang, M.; 1998: Zerkleinern und Stabilisieren von Tropfen beim mechanischen Emulgieren. Dissertation University of Karlsruhe, Fortschrittberichte VDI, Reihe 3, Nr. 527.

31. Ax, K., Schubert, H., Briviba, K., Rechkemmer, G., and Tevini, M.; 2001: Oil-in-water emulsions as carriers of bioavailable carotenoids. Proceedings PARTEC, 27.-29. March, Nuremberg/Germany. p. 1-8.

32. Engel, R. and Schubert, H.; 2005: Formulation of phytosterols in emulsions for increased dose response in functional foods. Innovative Food Science and Emerging Technologies 6, p. 233-237.

33. Ax, K.; 2004: Emulsionen und Liposomen als Trägersysteme für Carotinoide. Dissertation University of Karlsruhe, Shaker Verlag Aachen/Germany. 